\title{
MIMO RADAR WITH DIGITAL BEAMFORMING
}

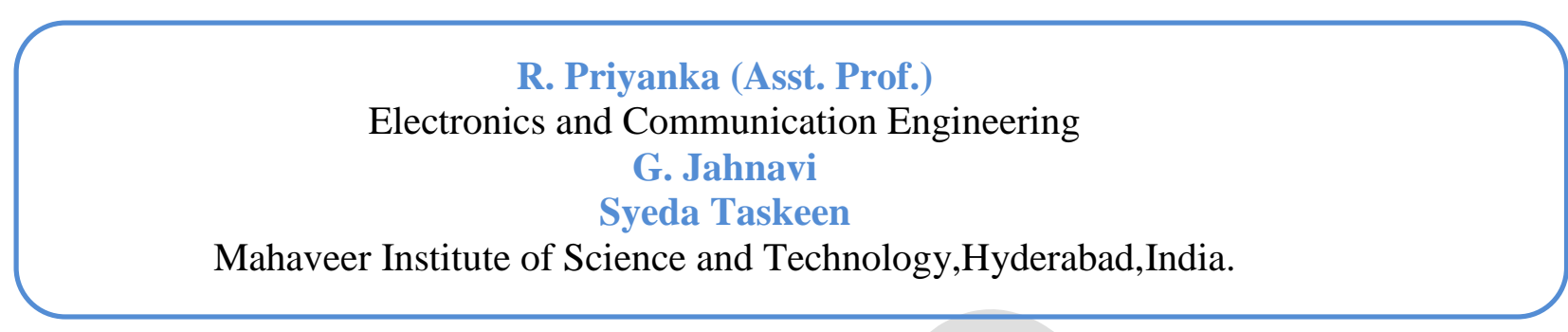

\section{ABSTRACT:}

The first radar has been patented 110 years ago. Meanwhile the applications became numerous and the system concepts have been adopted to the available technologies. Typical applications are speed control, air traffic control, synthetic aperture radar, airborne and space borne missions, military applications and remote sensing. Research for medical radar applications is well progressing for breast cancer detection and tumor localization. Automobile radar for save and autonomous driving are meanwhile produced in millions per year. In the next years the state-of-the-art radar system concepts will experience almost a revolution. Despite the significant advancements, the radar system technology did not develop like communications or other technologies during the last 20 years. Some of these new technologies will within a few years penetrate radar and revolutionize radar system concepts. This will then allow for new radar features and radar signal processing approaches.

\section{INTRODUCTION}

The first radar was patented in 1904 by Christian Hülsmeyer [1]. It was a pulsed radar, radiating differentiated video pulses, generated by a spark gap. Hülsmeyer's ideas were based on the experiments by Heinrich Hertz in 1888, when Hertz detected the polarization dependent reflection of electromagnetic waves. Since then radar system technology and signal processing have significantly been improved. The first electronically scanning radar was the German Search Radar FuMG 41/42 Mammut-1 in 1944. Numerous innovations in radar system technology followed since then, e.g. the FMCW radar technology. 
An important step was the invention of imaging Synthetic Aperture Radar (SAR) which was first introduced in 1956 by the patent of Carl Wiley [2] and the first civilian1 spaceborne SAR Seasat in 1978. In the same way the radar hardware technology and the radar signal processing have advanced significantly. Until 1990 radar technology was always a little ahead of the communications technology. But with the advent of the wide spread mobile communications, this situation changed. Although radars became equipped with new semiconductor devices and signal processing technologies, the system-level radar concepts have remained the same since many years; these radars still:

transmit the identical signal during operation

$>$ transmit only one frequency band at a time (e.g. FMCW)

$>$ 'see' only a small area at a time (e.g. phased array)

$>$ scan mechanically (e.g. airport radar)

Most of the current state-of-the-art radars, except for some military radars, transmit an identical signal for the whole time of their operation. Functionality-wise, this is inefficient since the radar will be limited to a narrow field of operation, when there are many different tasks/scenarios that are encountered even for a single radar, e.g. near range/far range, tracking, low/high range resolution, etc. Regarding electronic warfare, the radiation of uncorrelated signals is a necessity, otherwise countermeasures are straight forward.

The frequency spectrum has become the most valuable resource in the world since 20 years ago, because it is strictly limited and is not transferable. As such it must be used as efficiently as possible. Technologies that exploit the spectrum for opportunistic spectrum usage i.e. cognitive radio, or dualfuntional systems i.e. radar-communication systems, are already being extensively researched to take full advantage of the limited spectrum.

Since the year 2000, the number of radars being used is rapidly increasing. The fastest growing market for radar applications is the automotive radar. It is foreseeable that within a few years there will be millions of radars on the roads, with many cars equipped with up to five different radar systems. Consequently, there will be a selective inoperability in these radar systems due to strong inter-system interferences. Interference within the same frequency band can be avoided if the radar signals are properly coded and are continuously changing for low cross-correlation, like in communications.

For scanning radars, the conventional method is still to use a narrow beam, which is either mechanically or electronically scanned (i.e. phased arrays). This approach of scanning a wide area for 
target detection is highly inefficient. Mechanical scanning is cheap, but slow; the phased array method is faster, but rather expensive. In both cases only one beam-width area is scanned at a time.

The development in SAR system mainly manifests itself in the utilization of refined processing techniques, which take into account (and mitigate) various geometrical and system error sources. Most advanced digital hardware is used for the signal processing. The main change in the instrument hardware is the incorporation of transmit/receive modules, which enable advanced SAR modes and techniques [3], but are accompanied by a number of disadvantages regarding complexity, cost and calibration [4].

The drawbacks of conventional radars mentioned above and some other deficiencies of the current state-of-the-art radars must be overcome in the next ten years. The potential strategies for future radar system concepts will include:

$>$ intelligent signal coding, e.g. OFDM, CDMA

$>$ MIMO Radar - multiple transmit and receive antennas

$>$ digital beam forming for a higher angular resolution with wide coverage without mechanical moving parts

$>$ array imaging, efficient systems, reduced size and cost

$>$ combination of radar and communication $(\operatorname{RadCom}) 2$

These new system technologies will cause a revolution in radar concepts. In addition to the technical features, these will also allow cost reduction of the systems, increase the efficiency and the development of smart radars.

In the following sections these points will be explained in more detail and summarized from the view of communications and other well-known technologies.

\section{RADAR SIGNAL CODING}

\section{A. Radar Signal Coding Requirements}

The basic requirement for future radars is to cover time and spectrum simultaneously, differently coded with each transmitted signal. This allows the compression of the received signal in the dimensions of time and frequency, thus increasing the compression gain significantly, up to $50 \mathrm{~dB}-70 \mathrm{~dB}$. This in turn allows the reduction of the transmit power due to the additional compression gain. For small radars, e.g. automotive radars, this increases their power efficiency. For military applications the detectability of these radar signals for localization and countermeasures becomes much more difficult, because they are similar to communications signals. 
From communications several different coding schemes are known, e.g. CDMA (Code Division Multiple Access), DSSS (Direct Sequence Spread Spectrum), OFDM (Orthogonal Frequency Division Multiplexing) among others. For radar applications the signal model selection must be made according to (but not exclusively) [5][6]:

$>$ simplicity of signal generation

$>$ good decorrelation of simultaneous and consecutive signals

$>$ simple signal processing, ease of signal compression

$>$ possibility to transmit information

$>$ suitability for MIMO operation

$>$ simplicity of hardware realization

This list is longer for special applications such as missile control or ground penetrating radar. There is not one solution for all scenarios; hence in this paper the OFDM coding is selected, because it covers several of the above arguments, like simple realization, good decorrelation and simple processing.

\section{B. OFDM Radar Signal Coding}

The OFDM signal coding is well known from communications. The available and/or required spectrum is covered by multiple orthogonal subcarriers, which are all decorrelated -for short time shiftsdue to their pulse duration $T_{0}$ being inverse to the subcarrier distance $\Delta f$ :

$$
\Delta \mathrm{f}=1 / \mathrm{T}_{0}
$$

In Fig. 1 the OFDM carrier arrangement is shown. The decorrelation results, because the peak of a particular subcarrier coincides with the nulls of other subcarriers. A number of OFDM carrier modulations such as QAM (Quadrature Amplitude Modulation) and PSK (Phase Shift Keying) can be used. Arbitrary information such as music or data can then be modulated on the subcarriers. The spacing of the subcarriers is usually determined by the maximum expected Doppler shift $f_{\mathrm{D}}$ max. By the rule of thumb the subcarrier spacing should be more than 10 times the Doppler frequency to mitigate the effect of inter-carrier interference during the radar processing. The number of subcarriers $N_{\mathrm{C}}$ result from the subcarrier spacing $\Delta f$ related to the available bandwidth $B=N_{\mathrm{C}}$ 回 $\Delta f$. 


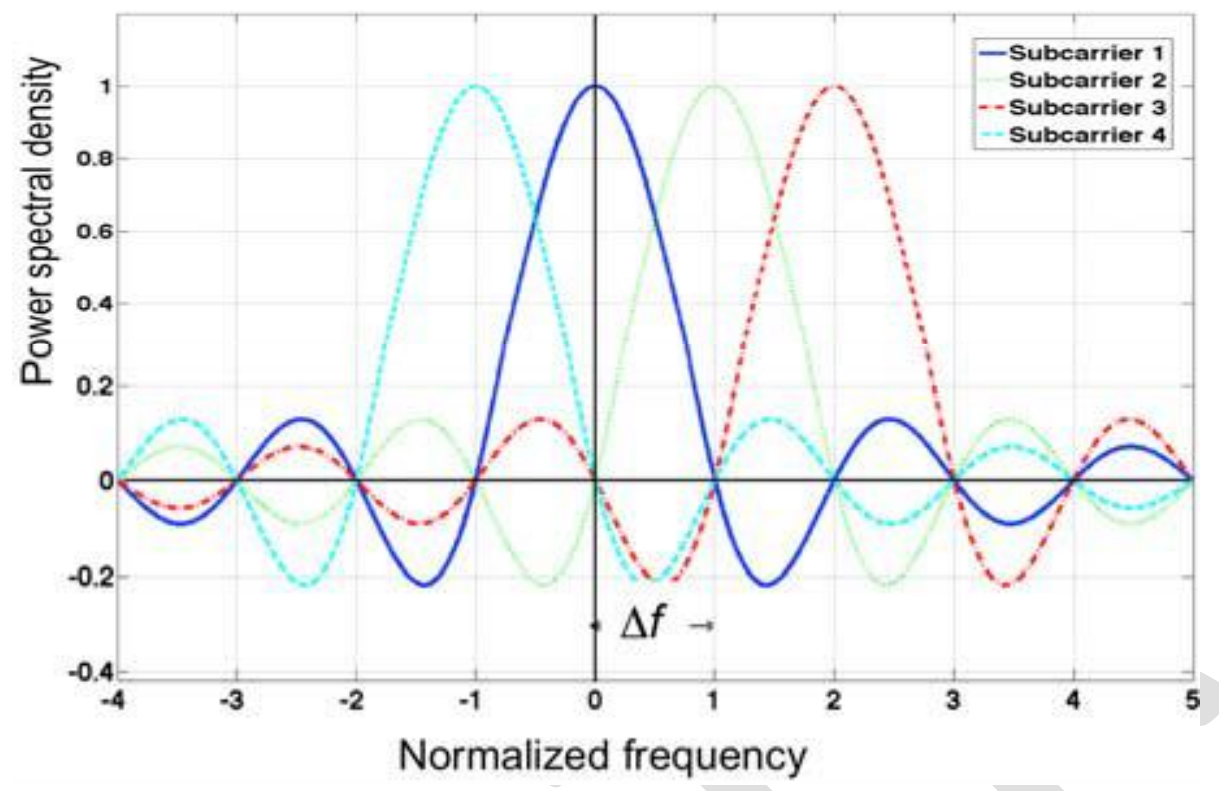

Fig. 1. Example of OFDM radar signal coding.

The total length of the transmit signal is composed of the number of carriers $N_{\mathrm{C}}$ and the number of consecutive symbols $M_{\mathrm{sym}}$ in time, which together form the transmit pulse. The signal simultaneously covers the whole bandwidth during the transmission interval $M_{\text {sym }} T 0$. In Fig. 2 the transmitted "block", as a matrix, is shown.

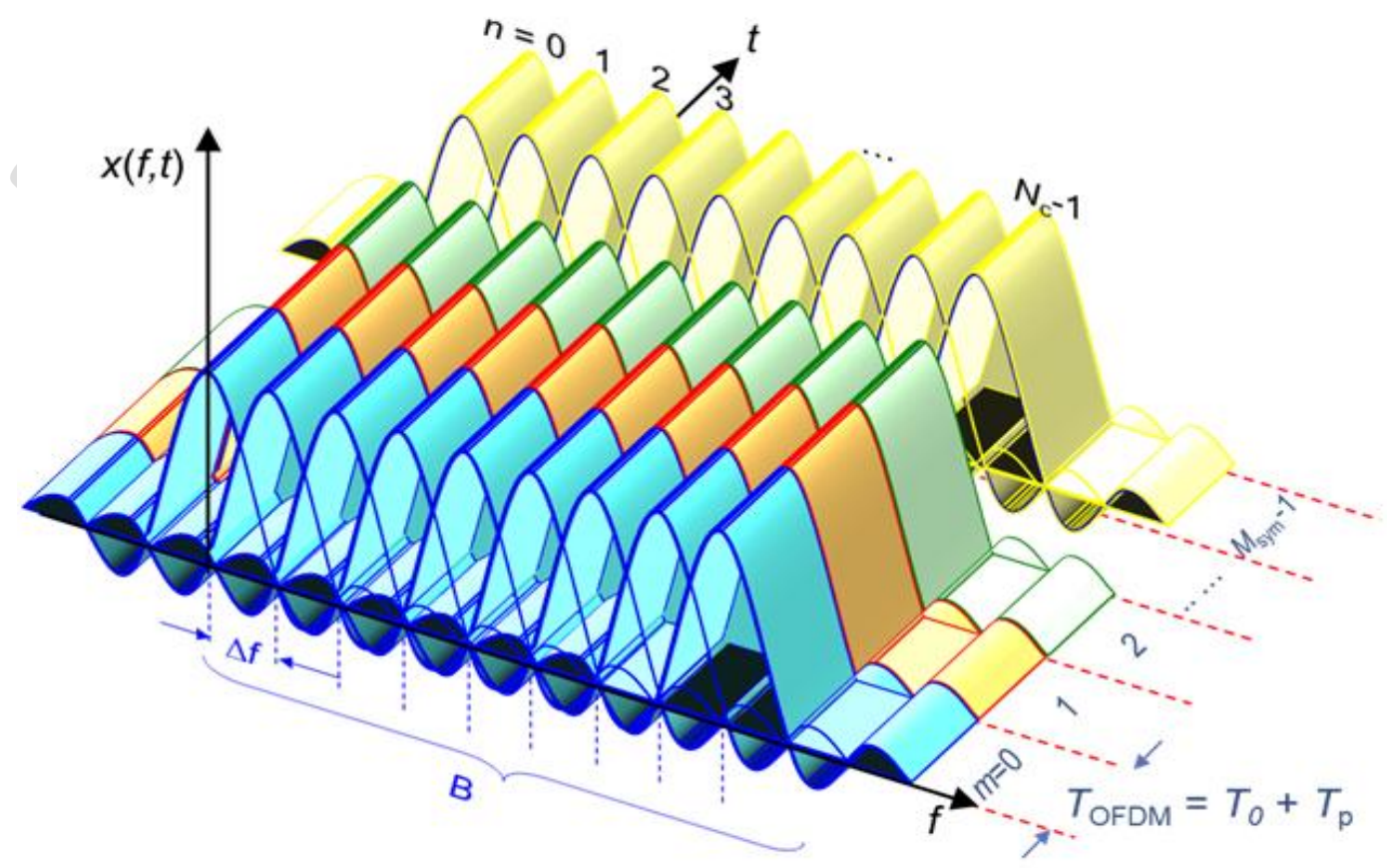

Fig. 2. OFDM radar transmit matrix with $N_{\mathrm{C}}$ carriers und $M_{\text {sym }}$ symbols. 
The transmitted signal is a matrix $\mathbf{D}(m, n)$, its elements are given by:

$$
\mathrm{X}(\mathrm{t})=\mathrm{A}_{\mathrm{Tx}}(\mathrm{m}, \mathrm{n}) \sum_{m=0}^{m s y m-1} \quad \sum_{n=0}^{\mathrm{Nc}-1} \mathrm{dTx}(\mathrm{mNc}+\mathrm{n}) \exp (\mathrm{j} 2 \pi \mathrm{fnt}) \cdot \operatorname{rect}(\mathrm{t}-\mathrm{mT} 0 / \mathrm{T} 0)
$$

With $m T_{0} \leq t \leq(m \square+1) T_{0}$

The radar processing steps will be discussed in another section. It will become apparent then that the transmitted information influences the outcome of the radar processing if a direct correlation is used to extract the range and Doppler information. This can be circumvented by using a Fourier based processing in [7]. As can be seen from Fig. 2, the total signal compression gain results from the multiplication of the compression in time and frequency domain. This results in the high compression gain mentioned earlier.

\section{DIGITAL BEAMFORMING}

Radars illuminate an angular area defined through the horizontal and vertical antenna beamwidths at any time. For operation over wider angular areas some sort of scanning either mechanically or electronically (i.e. beam switching or a phased array switching) is required. Conventional analog methods limit the effectiveness of scanning radars. A solution to this is Digital Beamforming (DBF). The basic idea of DBF is to transmit/receive multiple independent weighted beams formed by an array of antenna elements. The received signals of each antenna element are then down converted for A/D conversion and stored in a memory. From the memory an arbitrary number of beams can be digitally processed simultaneously, the basic operation can be thought of as a multiplication by a complex weight wi matrix. The major advantage is that the large elements' beam coverage can be simultaneously processed to multiple beams. The physical resolution is however still determined by the receive antenna basis $a \mathrm{Rx}$. Fig. 3 shows the principle of the received signal processing. 


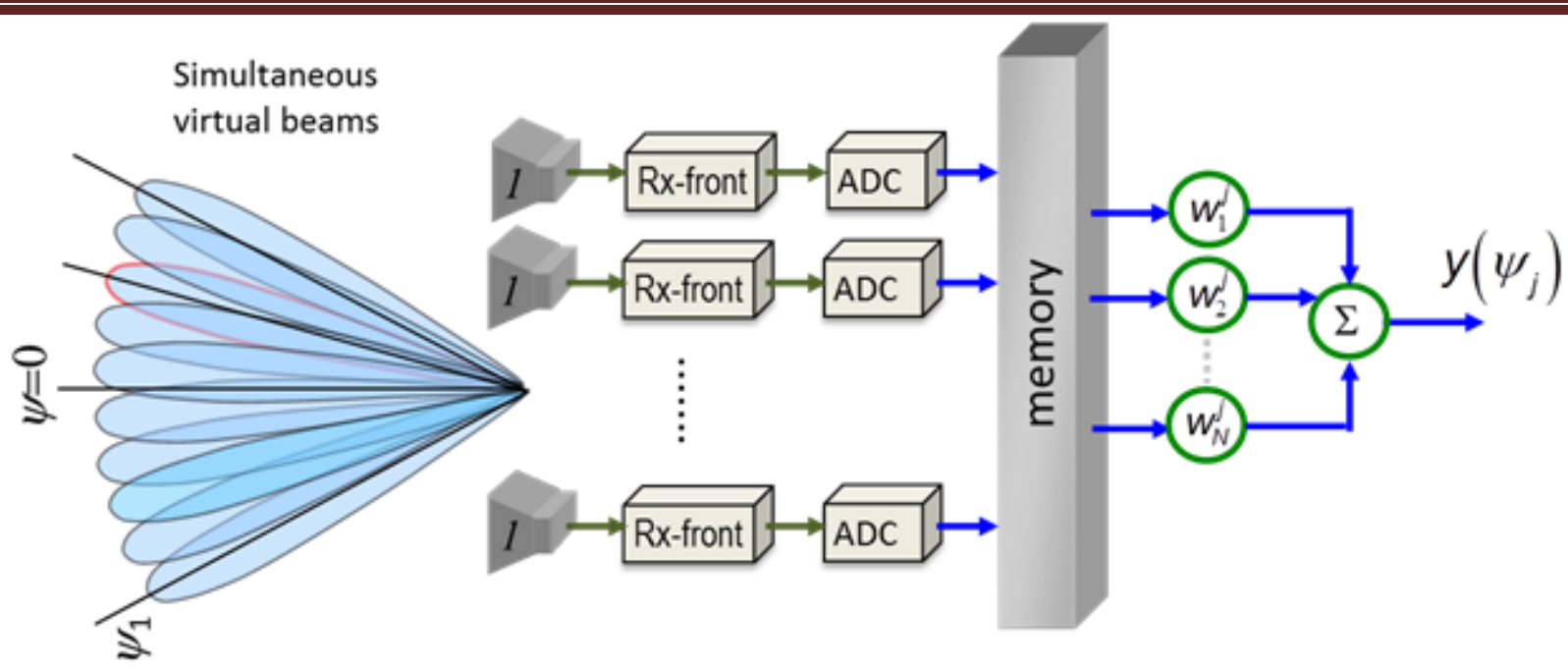

Fig. 3. The principle of digital beamforming.

\section{MIMO RADAR}

MIMO stands for Multiple-Input-Multiple-Output, a technology that is coming up in communications in order to improve the coverage, data rate and/or signal quality. For the future radar the same improvements are needed. MIMO radars simultaneously radiate uncorrelated signals, for instance, in different directions, or in the same direction with orthogonal polarizations [7][8][13][14]. This improves the coverage and the received information quality. An example for the radiation of three simultaneous beams with a MIMO radar is shown in Fig. 4. The decorrelation between the transmitted signals is realized by OFDM spectral-interleaving [15]. In this case the total bandwidth for each transmitreceive channel is retained hence there is no degradation in the radar range resolution, but the total radiated power is also interleaved, what means shared. This can be fully controlled. The radiation may be via different sub-arrays or via the same array.

The decorrelation of each transmit signal is important, otherwise small, remote targets might not show up on the radar image. In practice a decorrelation of more than $70 \mathrm{~dB}$ can be realized for OFDM interleaved signals. 


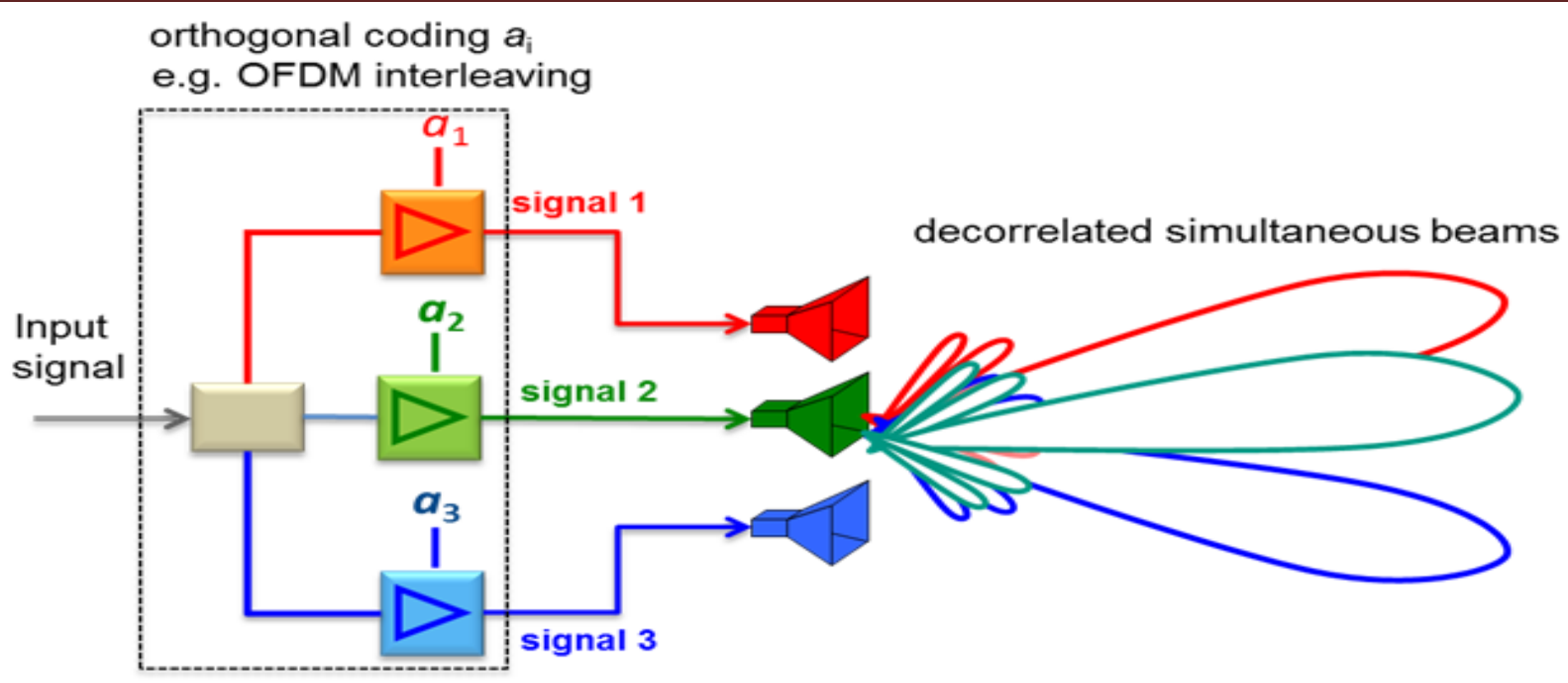

Fig. 4. MIMO transmitt array with three beams in different direction.

For near-range radar, like automotive radar, the coupling transmit-receive should be less than $40 \mathrm{~dB}$, because these radars usually transmit, while they receive the near range signals.

\section{MIMO SYNTHETIC APERTURE RADAR}

The main innovative characteristic of forthcoming generations of SAR systems is the use of multiple elevation and/or azimuth receiver channels combined with digital beamforming (DBF) capability [18][19]. This allows for the synthesis of multiple or dynamic digital receiver beams. Further, multiple transmit channels are being suggested as an extension to DBF systems. With this, we arrive at what is commonly referred to as MIMO SAR.

On a first view, MIMO SAR, does not seem to make sense. Indeed a prerequisite for a synthetic aperture is a moving platform, which suggests that the spatial position of an additional transmitter will be reached by a single moving transmitter after a time delay. Nevertheless, a more detailed view reveals that adding transmitters can be beneficial as it offers for example interferometric capabilities or simultaneous polarimetry [11].

The virtue of MIMO SAR is that it extends the dimensionality of the trade space [12]. This allows the conception of new systems, which overcome the "fundamental limitation" of conventional SAR. A good example is the simultaneous High-azimuth-Resolution and Wide-Swath SAR also known as HRWS, which improves two imaging parameters without sacrificing others. It is comprehensible that multichannel SAR allows innovative, and new combinations of operation techniques (modes). 


\section{RADAR 2020 SYSTEM BLOCK DIAGRAM}

The integration of a MIMO radar with DBF, operated with OFDM signals is shown in Fig. 5. The Radar 2020 system is completely digital except for the frontends. These frontends are identical on the transmit and receive side, respectively, for each channel. For higher frequencies and shorter ranges, e.g. in automotive applications, they may be completely integrated in MMICs. OFDM MIMO signal generators are already available on the market for certain applications. The OFDM parameters can then be set according to the application requirements.

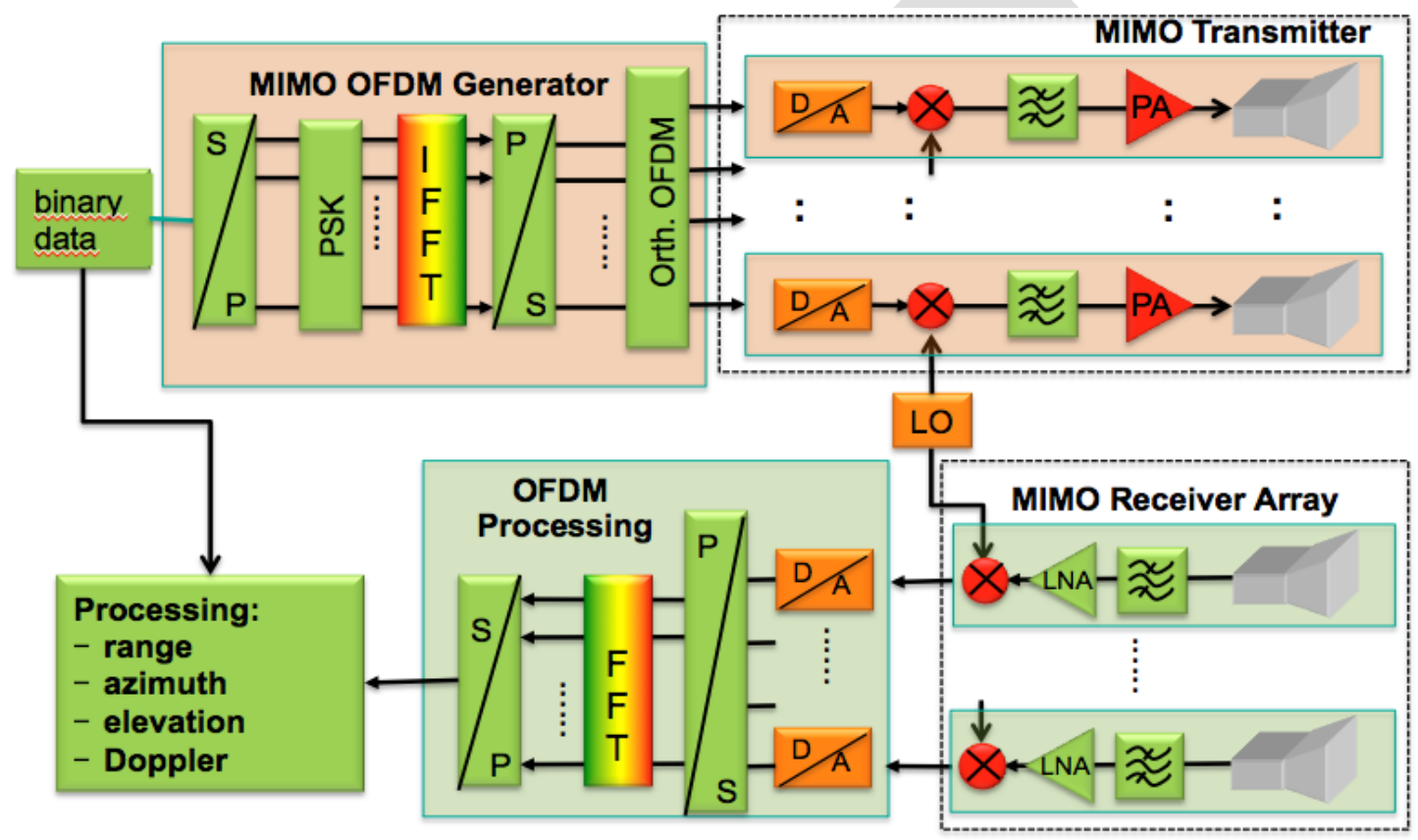

Fig. 5. Block diagram of the Radar 2020 with OFDM coding.

\section{RADAR SIGNAL PROCESSING}

The processing strategy for future radar systems in this paper is limited to OFDM. The transmit signal is given by equation (2), the receive signal $y(t)$ varies in amplitude, range and Doppler, see equation (7).

$$
\mathrm{y}(\mathrm{t})=\sum_{m=0}^{m s y m-1} \quad \sum_{n=0}^{\mathrm{Nc}-1} \mathrm{a}(\mathrm{m}, \mathrm{n}) \mathrm{dTx}(\mathrm{mNc}+\mathrm{n}) \exp \left(\mathrm{j} 2 \pi \mathrm{fn}\left(\mathrm{t}-\frac{2 \mathrm{R}}{2}\right)\right) \cdot \exp (\mathrm{g} 2 \pi \mathrm{fdt}) \operatorname{rect}(\mathrm{t}-\mathrm{mT} 0 / \mathrm{T} 0)
$$

For the evaluation of the reflected signals the information content in the OFDM receive signal is discarded via an element-wise division of the received signal by the transmit signal. What is left then are amplitude, time shift (range) and frequency shift (Doppler). The resulting equation (8) has a linear dependency with $n(f \mathrm{n})$ and $m(f \mathrm{D})$. 


$$
\mathrm{y}(\mathrm{t})=\sum_{m=0}^{m \text { sym }-1} \quad \sum_{n=0}^{\mathrm{Nc}-1} \mathrm{a}(\mathrm{m}, \mathrm{n}) \exp (\mathrm{j} 2 \pi \mathrm{fnt}) \cdot \exp (\mathrm{l}-\mathrm{j} 2 \pi \mathrm{f} 2 \mathrm{R} / \mathrm{C} 0) \exp (\mathrm{j} 2 \pi \mathrm{fdm} T \mathrm{Tofdm})
$$

From the extracts of equation (8) the range and Doppler can be determined by simple Fourier transformations (FFT, IFFT). In summary the angular DBF, range and Doppler processing are all just Fourier transformations. Simulations and experiments have proved that an unlimited number of targets can be resolved [9], limited only by the physical constraints such as the bandwidth $B$ and receive power $P \mathrm{Rx}$. The compression gain is the product of $M \cdot N$. High angular resolution algorithms like MUSIC can also be applied to generate an angular pseudo-spectrum. The overall efficiency of the Radar 2020 will in practice be $6 \mathrm{~dB}$ to $10 \mathrm{~dB}$ higher than for the current radars; this can be invested in wider beams for simultaneous coverage of areas of interest, for example for airport radar.

\section{CONCLUSION}

The innovative system technologies of the Radar 2020 will allow complete new functions and applications, which can replace most of the existing system concepts [16]. The radar of the future will render more information, be more flexible and will also be smaller and significantly cheaper. Best of all most of these technologies for the future radar systems are already available from other applications; they just have to be integrated in the future radars. The Radar 2020 will revolutionize radar system engineering, and foreseeably, also the radar market.

\section{REFERENCES}

[1] Ch. Hülsmeyer, "Verfahren um entfernte metallische Gegenstände mittels elektrischer Wellen einem Beobachter zu melden,” Kaiserliches Patentamt, Patentschrift Nr. 165546, 30. April 1904.

[2] C. A. Wiley, "Pulsed Doppler radar methods and apparatus," U.S. Patent 3.196.436, 1954.

[3] M. Ludwig, H.-P. Feldle, and H. Ott, “A miniaturised X-band T/Rmodule for SAR-systems based on active phased array techniques," in Proc. Int. Geoscience and Remote Sensing Symposium IGARSS'95, vol 3, 1955, pp. 2063-2065.

[4] M. Younis, W. Wiesbeck, "SAR with digital beamforming on receive only," Proc. Int. Geoscience and Remote Sensing Symposium IGARSS’99, Jun 1999, pp. 1773-1775.

[5] B. Liu, Z. He, J. Zeng, and B. Liu, "Polyphase orthogonal code design for MIMO radar systems," in Proc. Int. Conf. Radar (CIE), Oct. 2006, pp. 1-4. 
[6] C. Sturm and W. Wiesbeck, "Waveform Design and Signal Processing Aspects for Fusion of Wireless Communications and Radar Sensing", Proceedings of the IEEE, vol. 99, pp. 1236-1259, July 2011.

[7] A. Hassanien and S. A. Vorobyov, "Transmit/receive beamforming for MIMO radar with colocated antennas", Proc. IEEE Intl. Conf. Acoust., Speech, and Signal Proc., Taipei, 2009.

[8] B. Friedlander, "On the relationship between MIMO and SIMO radars", IEEE Trans. Signal Processing, pp. 394-398, Jan., 2009. [9] Y. L. Sit, L. Reichardt, C. Sturm, and T. Zwick, "Verification of an OFDM-based Range and Doppler Estimation Algorithm with Ray-

Tracing", in International Conference on Electromagnetics in Advanced Applications (ICEAA '11), Turin, Italy, Sep. 2011.

[10] Y. L. Sit, C. Sturm, and T. Zwick, "Interference Cancellation for Dynamic Range Improvement in an OFDM Joint Radar and Communication System", in European Radar Conference (EuRAD), Manchester, UK, Oct. 2011.

[11] G. Krieger, N. Gebert, and A. Moreira, "Multidimensional waveform encoding: A new digital beamforming technique for synthetic aperture radar romote sensing," in IEEE Trans. on Geoscience and Remote Sensing, vol 46, no. 1, pp. 31-46, Jan. 2008.

[12] M. Younis, P. López-Dekker, and G. Krieger, "MIMO SAR operation modes and techniques," in Proc. European Conference on Synthetic Aperture Radar EUSAR'2002, Köln, Germany, June 2002, pp. 187-190.

[13] J. Li and P. Stoica (editors), MIMO radar signal processing, John Wiley \& Sons, 2009.

[14] L. Reichardt, J. Pontes, L. Sit, and T. Zwick, "Antenna Optimization for Time-variant MIMO Systems", in Proceedings of the 5th European Conference on Antennas and Propagation, Rome, Italy, Apr. 2011.

[15] C. Sturm, Y. L. Sit, M. Braun, T. Zwick, "Spectrally Interleaved Multi- Carrier Signals for Radar Network Applications and MIMO-Radar“ IET Radar, Sonar \& Navigation, 2013

[16] W. Wiesbeck, "Radar of the Future," Proceedings of the 10th European Radar Conference, 9-11 Oct. 2013, Nuremberg, Germany, pp. 137-140.

[17] Y. Leen, C. Sturm, L. Reichhardt, T. Zwick, and W. Wiesbeck, "The OFDM joint radarcommunication system: An overview," in The Third International Conference on Advances in Satellite and Space Communications, 201 SPACOMM, 2011. 
[18] J.-H. Kim, M. Younis, A. Moreira, and W. Wiesbeck, "A Novel OFDM Chirp Waveform Scheme for Use of Multiple Transmitters in SAR”, IEEE Geoscience and Remote Sensing Letters, vol. 10, no. 3, May 2013, pp. 568-572.

[19] Krieger, G., "MIMO-SAR: Opportunities and Pitfalls," Geoscience and Remote Sensing, IEEE Transactions on , vol.52, no.5, pp.2628,2645, May 2014 